\title{
AN EXTENSION OF A THEOREM OF E. STEIN
}

\section{CONCEPCIÓN BALLESTER PEREYRA ${ }^{1}$}

Consider the singular integral

$$
(T f)(x)=\text { P.V. } \int_{E_{n}} \frac{H(x, x-y)}{|x-y|^{n}} f(y) d y,
$$

where $E_{n}$ is the $n$-dimensional Euclidean space, and the integral is taken in the Lebesgue sense.

Calderón and Zygmund, [2], [3], have proved that under suitable conditions on the kernel $H(x, x-y)$

$$
\|T f\|_{p} \leqq A\|f\|_{p}, \quad 1<p<\infty,
$$

where $A$ is a positive constant and $\|\cdot\|_{p}$ is the $L_{p}$ norm. In the onedimensional case Babenko [1] has shown that this inequality remains true if the measure $d x$ is replaced by the measure $|x|^{\beta} d x$, and E. Stein [4] proved that in $n$ dimensions

$$
\left\|(T f)(x)|x|^{\beta}\right\|_{p} \leqq A_{p, \beta}\left\|f(x)|x|^{\beta}\right\|_{p}
$$

for $1<p<\infty$ and $-n / p<\beta<n / p^{\prime},\left(1 / p+1 / p^{\prime}=1\right)$.

He based his proof on the following

Lemma (Stein). Let

$$
K(x, y)=\left|1-(|x| /|y|)^{\beta}\right| /|x-y|^{n}
$$

and let

$$
(U f)(x)=\int_{E_{n}} K(x, y) f(y) d y .
$$

Then

$$
\|(U f)(x)\|_{p} \leqq A_{p, \beta}\|f(x)\|_{p} \quad \text { if }-n / p<\beta<n / p^{\prime} .
$$

Stein's proof of (3) does not apply to $p=1$. In fact, the operator $T$ is not of type $(1,1)$, i.e. it does not satisfy $(2)$ for $p=1$. But $T$ is of weak type $(1,1)$, that is, for some $s<1$, and every set $X$ of finite measure

Received by the editors May 16, 1967.

${ }^{1}$ Present address: Departamento de Matemáticas, Facultad de Ciencias, Universidad Central de Venezuela, Caracas. 
(6)

$$
\left(\int_{X}|(T f)(x)|^{s} d x\right)^{1 / s} \leqq M|X|^{(1 / 8)-1}\|f\|_{1}
$$

where $M$ is a positive constant, and $|X|$ is the Lebesgue measure of $X$. We will prove

Theorem 1. Let $-n<\beta<0$, and let Uf be defined by (5). Then Uf is of weak type $(1,1)$.

And, as a consequence,

THEOREM 2. Let

$$
(T f)(x)=\text { P.V. } \int_{E_{n}} \frac{H(x, x-y)}{|x-y|^{n}} f(y) d y
$$

and assume that $T$ is of weak type (1.1), and that $|H(x, x-y)| \leqq B$. Then

$$
\left(\int_{X}|(T f)(x)|^{\mid s}|x|^{\mid s} d x\right)^{1 / 8} \leqq M|x|^{(1-8) / 8}|f(x)| x \mid \beta \|_{1}
$$

for every set $X$ of finite measure, $-n<\beta<0$ and $(n-1) / n<s<1$.

Proof of Theorem 1 . We will prove that $U$ satisfies (6) for any $s$ such that

$$
(n-1) / n<s<1 .
$$

Let $\lambda=|y| /|x|$. The kernel $K(x, y)$ is singular for $\lambda=0, \lambda=1, \lambda=\infty$. Define:

$$
\begin{array}{ll}
K_{1}(x, y)=K(x, y) & \text { for } 0 \leqq \lambda \leqq 1 / 2, \text { zero otherwise, } \\
K_{2}(x, y)=K(x, y) & \text { for } \lambda \geqq 2, \text { zero otherwise, and } \\
K_{3}(x, y)=K(x, y) & \text { for } 1 / 2<\lambda<2, \text { zero otherwise, }
\end{array}
$$

and set

$$
\left(U_{i} f\right)(x)=\int_{E_{n}} K_{i}(x, y) f(y) d y, \quad i=1,2,3,
$$

assuming, as we may, $f(x) \geqq 0$. Let $\xi$ be the unit vector in the direction of $x, r=|x|, \eta$ the unit vector in the direction of $y$ and $R=|y|$. Let $d \omega_{\xi}$ and $d \omega_{\eta}$ be the elements of Euclidean measure on the spheres $|x|=1$ and $|y|=1$, and call $\Sigma_{\xi}$ and $\Sigma_{\eta}$ those unit spheres. Then (8) can be written 


$$
\left(U_{\mathrm{i}} f\right)(x)=\int_{\Sigma_{\eta}} \int_{0}^{\infty} K_{i}(r \xi, R \eta) f(R \eta) R^{n-1} d R d \omega_{\eta} .
$$

$K(x, y)$ is homogeneous of order $-n$, so the change of variable $R=\lambda r$ gives

$$
\left(U_{i} f\right)(x)=\int_{\Sigma_{\eta}} \int_{0}^{\infty} K_{i}(\xi, \lambda \eta) f(\lambda r \eta) \lambda^{n-1} d \lambda d \omega_{\eta} .
$$

For $i=1$, we have

$$
K_{1}(\xi, \lambda \eta) \leqq \frac{\left|1-\lambda^{-\beta}\right|}{\left|1-2 \lambda \cos (\xi, \eta)+\lambda^{2}\right|^{n / 2}} \leqq A\left|1-\lambda^{-\beta}\right|
$$

because $\left[1-2 \lambda \cos (\xi, \eta)+\lambda^{2}\right]^{n-2} \geqq A>0$ if $0 \leqq \lambda \leqq 1 / 2, A$ being a constant. Therefore

$$
\left|\left(U_{1} f\right)(r \xi)\right| \leqq A \int_{\Sigma_{\eta}} \int_{0}^{1 / 2}\left|1-\lambda^{-\beta}\right| \lambda^{n-1} f(\lambda r \eta) d \lambda d \omega_{\eta} .
$$

We need an estimate for the integral

$$
\left[\int_{X}\left|\left(U_{1} f\right)(x)\right|^{s} d x\right]^{1 / 8}=\left[\int_{\Sigma_{\xi}} \int_{X_{\xi}}\left|\left(U_{1} f\right)(r \xi)\right|{ }^{s} r^{n-1} d r d \omega_{\xi}\right]^{1 / s}
$$

where, for each $\xi$ in the unit sphere $\Sigma_{\xi}, X_{\xi}$ is the set of $r$ 's such that $r \xi \in X$. By (11) and (12)

$$
\begin{aligned}
& \left(\int_{X}\left|\left(U_{1} f\right)(x)\right|^{8} d x\right)^{1 / 8} \\
& \leqq A\left\{\int_{\Sigma_{\xi}}\left[\int_{X_{\xi}}\left(\int_{\Sigma_{\eta}} \int_{0}^{1 / 2}\left|1-\lambda^{-\beta}\right| \lambda^{n-1} f(\lambda r \eta) d \lambda d \omega_{\eta}\right)^{s} \cdot \cdot r^{n-1} d r\right] d \omega_{\xi}\right\}^{1 / 8} \\
& =A\left\{\int _ { \Sigma _ { \xi } } \left[\int_{X \xi}\left(\int_{\Sigma_{\eta}} \int_{0}^{1 / 2}\left|1-\lambda^{-\beta}\right| \lambda^{n-1} f(\lambda r \eta) r^{(n-1) / 8} d \lambda d \omega_{\eta}\right)^{s}\right.\right. \\
& \left.\cdot d r] d \omega_{\xi}\right\}^{1 / 8}
\end{aligned}
$$

and by Hölder inequality, since $s<1$, 


$$
\begin{aligned}
& \left(\int_{X}\left|\left(U_{1} f\right)(x)\right|^{8} d x\right)^{1 / s} \\
& \leqq A\left\{\int_{\Sigma_{\xi}}\left[\int_{X_{\xi}} \int_{\Sigma_{\eta}} \int_{0}^{1 / 2}\left|1-\lambda^{-\beta}\right| \lambda^{n-1} f(\lambda r \eta) r^{(n-1) / 8} d \lambda d \omega_{\eta} d r\right]^{8}\right. \\
& \left.\cdot\left[\int_{X_{\xi}} d r\right]^{1-8} d \omega_{\xi}\right\}^{1 / s} .
\end{aligned}
$$

Since $0 \leqq \lambda \leqq 1 / 2, \beta<0$, then $\left|1-\lambda^{-\beta}\right| \leqq 1$, and setting $l=\lambda r$ the above yields

$$
\begin{aligned}
& \left(\int_{X}\left|\left(U_{1} f\right)(x)\right|^{s} d x\right)^{1 / 8} \\
& \leqq A\left\{\int_{\Sigma_{\xi}}\left[\int_{X_{\xi}} \int_{\Sigma_{\eta}} \int_{0}^{\infty} l^{n-1} f(l \eta) \frac{r^{(n-1) / 8}}{r^{n-1}} \frac{d l}{r} d \omega_{\eta} d r\right]^{s}\right. \\
& \left.\cdot\left|X_{\xi}\right|^{1-s} d \omega_{\xi}\right\}^{1 / s},
\end{aligned}
$$

where we have replaced the in tegral $\int_{x_{\xi}} d r$ by its value $\left|X_{\xi}\right|$. But the variables in the brackets are separated, so the right side of (13) is

$$
A\left\{\int_{\Sigma_{\xi}}\left[\int_{X_{\xi}} r^{(n-1-n s) / s} d r\right]^{s}\left[\int_{\Sigma_{\eta}} \int_{0}^{\infty} l^{n-1} f(l \eta) d l d \omega_{\eta}\right]^{s}\left|X_{\xi}\right|^{1-s} d \omega_{\xi}\right\}^{1 / 8} .
$$

But since $\int_{\Sigma_{\eta}} \int_{0}^{\infty} l^{n-1} f(l \eta) d l d \omega_{\eta}=\| f_{\| 1}$,

$$
\begin{aligned}
\left\{\int_{X}\left|\left(U_{1} f\right)(x)\right| s d x\right\}^{1 / 8} & \\
& \leqq A\|f\|_{1}\left\{\int_{\Sigma_{\xi}}\left|X_{\xi}\right|^{1-s}\left[\int_{X_{\xi}} r^{(n-1-n s) / 8} d r\right]^{8} d \omega_{\xi}\right\}^{1 / 8} .
\end{aligned}
$$

$X$ being a set of finite measure, $X_{\xi}$ can be infinite only in a set of measure zero. Thus, since by (7),

$$
-1<(n-1-n s) / s<0
$$

the integral in the inner bracket of (14) is convergent, and as the power of $r$ is negative, it does not exceed

$$
\int_{0}^{\left|X_{\xi}\right|} r^{(n-1-n s) / 8} d r=s /(n-1-n s+s)\left|X_{\xi}\right|^{(n-1-n s+8) / \bullet} .
$$

Replacing in (14), and applying Hölder's inequality once more, 


$$
\begin{aligned}
& \left(\int_{X}\left|\left(U_{1} f\right)(x)\right|^{8} d x\right)^{1 / 8} \\
& \quad \leqq A_{1}\|f\|_{1}\left\{\int_{\Sigma \xi}\left|X_{\xi}\right|^{1-s}\left(\left|X_{\xi}\right|^{(n-1-n s+s) / 8)} d \omega_{\xi}\right\}^{1 / 8}\right. \\
& \quad=A_{1}\|f\|_{1}\left\{\int_{\Sigma \xi}\left|X_{\xi}\right|^{n(1-s)} d \omega_{\xi}\right\}^{1 / 8} \\
& \quad \leqq A_{1}\|f\|_{1}\left(\int_{\Sigma \xi}\left|X_{\xi}\right|^{n} d \omega_{\xi}\right)^{(1-s) / 8}\left(\int_{\Sigma \xi} d \omega_{\xi}\right) \\
& \leqq M_{1}\|f\|_{1}|X|(1-s) / 8
\end{aligned}
$$

Consider now $U_{2} f$. Here $2 \leqq \lambda<\infty$, so

(16) $\left(U_{2} f\right)(r \xi)=\int_{\Sigma \eta} \int_{2}^{\infty} \frac{\left|1-\lambda^{-\beta}\right| \lambda^{n-1}}{\left|1-2 \lambda \cos (\xi, \eta)+\lambda^{2}\right|^{n / 2}} f(\lambda r \eta) d \lambda d \omega$.

In this case we get

$$
\frac{\left|1-\lambda^{-\beta}\right|}{\left|1-2 \lambda \cos (\xi, \eta)+\lambda^{2}\right|^{n / 2}} \leqq B \frac{\left|1-\lambda^{-\beta}\right|}{\lambda^{n}} .
$$

We set

$$
I=\left\{\int_{X}\left|\left(U_{2} f\right)(x)\right|^{s} d x\right\}^{1 / s}=\left\{\int_{\Sigma \xi} \int_{X \xi}\left|\left(U_{2} f\right)(r \xi)\right|^{s} r^{n-1} d r d \omega_{\xi}\right\}^{1 / s} .
$$

Using (17) in (16) and replacing above,

$$
\begin{aligned}
& I \leqq B\left\{\int_{\Sigma_{\xi}}\left[\int_{X_{\xi}}\left(\int_{\Sigma_{\eta}} \int_{2}^{\infty} \frac{\left|1-\lambda^{-\beta}\right|}{\lambda} f(\lambda r \eta) d \lambda d \omega_{\eta}\right)^{s} r^{n-1} d r\right] d \omega_{\xi}\right\}^{1 / s} \\
&=B\left\{\int_{\Sigma_{\xi}} \int_{X_{\xi}}\left(\int_{\Sigma_{\eta}} \int_{2}^{\infty} \frac{\left|1-\lambda^{-\beta}\right|}{\lambda} f(\lambda r \eta) r^{(n-1-\epsilon) / 8} d \lambda d \omega_{\eta}\right)^{s}\right. \\
&\left.\left.\cdot r^{\epsilon} d r\right] d \omega_{\xi}\right\}^{1 / s},
\end{aligned}
$$

and by Hölder's inequality 


$$
\begin{aligned}
I \leqq B\left\{\int_{\Sigma_{\xi}}\left[\int_{X_{\xi}} \int_{\Sigma_{\eta}} \int_{2}^{\infty} \frac{\left|1-\lambda^{-\beta}\right|}{\lambda} f(\lambda r \eta) r^{(n-1-\epsilon) / 8} d \lambda d \omega_{\eta} d r\right]^{s}\right. \\
\left.\cdot\left[\int_{X_{\xi}} r^{\epsilon /(1-8)} d r\right]^{1-8} d \omega_{\xi}\right\}^{1 / 8},
\end{aligned}
$$

which by the change of in tegration variable $\rho=r \lambda$, becomes

$$
\begin{aligned}
I \leqq B\left\{\int_{\Sigma_{\xi}}\left[\int_{0}^{\infty} \int_{\Sigma_{\eta}} \int_{2}^{\infty} \frac{\left|1-\lambda^{-\beta}\right|}{\lambda} f(\rho \eta) \frac{\rho^{(n-1-\epsilon) / 8}}{\lambda^{(n-1-\epsilon) / 8}} d \lambda d \omega_{\eta} \frac{d \rho}{\lambda}\right]^{s}\right. \\
\left.\cdot\left[\int_{X_{\xi}} r^{\epsilon /(1-s)} d r\right]^{1-8} d \omega_{\xi}\right\}^{1 / 8} .
\end{aligned}
$$

In the first bracket the variables are separated so the right member above can be given the form

$$
\begin{aligned}
B\left\{\int _ { \Sigma _ { \xi } } ( \int _ { X \xi } r ^ { \epsilon / ( 1 - s ) } d r ) ^ { 1 - \delta } \left(\int_{0}^{\infty}\right.\right. & \left.\int_{\Sigma_{\eta}} f(\rho \eta) \rho^{(n-1-\epsilon) / 8} d \omega_{\eta} d \rho\right)^{8} \\
& \left.\left(\int_{2}^{\infty} \frac{\left|1-\lambda^{-\beta}\right|}{\lambda^{2+(n-1-\epsilon) / 8}} d \lambda\right)^{s} d \omega_{\xi}\right\}^{1 / 8} .
\end{aligned}
$$

Recalling (18) and noticing $\int_{0}^{\infty} \int_{\Sigma_{\eta}} f(\rho \eta) \rho^{n-1} d \omega_{\eta} d \rho=\|f\|_{1}$, we obtain

$$
I \leqq B\|f\|_{1}\left\{\int_{\Sigma \xi}\left(\int_{X_{\xi}} r^{n-1} d r\right)^{1-8}\left(\int_{2}^{\infty} \frac{\left|1-\lambda^{-\beta}\right|}{\lambda^{n+1}} d \lambda\right)^{8} d \omega_{\xi}\right\}^{1 / 8} .
$$

As $\beta>-n$, the integral with regard to $\lambda$ is convergent, and

$$
\begin{aligned}
\left(\int_{X}\left|\left(U_{2} f\right)(x)\right|^{8} d x\right)^{1 / 8} & \leqq \text { Const }\|f\|_{1}\left[\int_{\Sigma_{\xi}}\left(\int_{X_{\xi}} r^{n-1} d r\right)^{1-8} d \omega_{\xi}\right]^{1 / 8} \\
& \leqq \text { Const }\|f\|_{1}\left\{\left(\int_{\Sigma_{\xi}} \int_{X \xi} r^{n-1} d r d \omega_{\xi}\right)^{1-8}\left(\int_{\Sigma \xi} d \omega_{\xi}\right)^{\bullet}\right\}^{1 / 8}
\end{aligned}
$$

or

$$
\left[\int_{X}\left|\left(U_{2} f\right)(x)\right|^{s} d x\right]^{1 / s} \leqq M_{2}\|f\|_{1}|X|^{(1-s) / s} .
$$

In the case of $U_{3}$, Stein's proof applies, and $U_{3}$ is $L_{1}$ bounded, and a fortiori of weak type $(1,1)$. Thus with $(15)$ and $(21)$ the inequality (6) is established. 
Proof of Theorem 2. By hypothesis

$$
\left\{\int_{X}\left|T\left[f(y)|y|^{\beta}\right]\right|^{s} d x\right\}^{1 / s} \leqq M_{1}|x|^{(1-8) / s}\|f(y)|y| \beta\|_{1} .
$$

It suffices to prove that

$$
\left.\left.\left.\int_{X}\left|T\left[f(y)|y|^{\beta}\right]-\right| x\right|^{\beta} T[f(y)]\right|^{s} d x\right\}^{1 / s} \leqq M_{1}|X|(1-s) / s|| f(y)|y| \beta \|_{1} .
$$

We have

$$
\begin{aligned}
\mid T & {\left[f(y)|y|^{\beta}\right]-|x|^{\beta} T[f(y)] \mid } \\
& =\left.\left|\int_{E_{n}} \frac{H(x, x-y)}{|x-y|^{n}} f(y)\right| y\right|^{\beta} d y-\int_{E_{n}} \frac{H(x, x-y)}{|x-y|^{n}} f(y)|x|^{\beta} d y \mid \\
& =\left|\int_{E_{n}} H(x, x-y) \frac{|y|^{\beta}-|x|^{\beta}}{|x-y|^{n}} f(y) d y\right| \\
& \leqq C \int_{E_{n}} \frac{\left.|1-| x\right|^{\beta} /|y|^{\beta} \mid}{|x-y|^{n}}|y|^{\beta} f(y) d y \\
& =C \int_{E_{n}} K(x, y)|y|^{\beta} f(y) d y .
\end{aligned}
$$

An application of Theorem 1 now yields the desired result.

\section{REFERENCES}

1. K. I. Babenko, On conjugate functions, Dokl. Akad. Nauk SSSR 62 (1948), 157-160.

2. A. P. Calder6n and A. Zygmund, On the existence of certain singular integrals, Acta Math. 88 (1952), 85-139.

3. - On singular integrals, Amer. J. Math. 78 (1956), 289-309.

4. E. Stein, Note on singular integrals, Proc. Amer. Math. Soc. 8 (1957), 250-254.

UNIVERSITY OF WisCONSIN 